\title{
IL 10A genotypic association with decreased IL-10 circulating levels in malaria infected individuals from endemic area of the Brazilian Amazon
}

Virginia A Pereira', Juan C Sánchez-Arcila ${ }^{1}$, Antonio Teva ${ }^{2}$, Daiana S Perce-da-Silva ${ }^{3}$, Mariana PA Vasconcelos ${ }^{4}$, Cleoni AM Lima ${ }^{5}$, Cesarino JL Aprígio ${ }^{6}$, Rodrigo N Rodrigues-da-Silva ${ }^{1}$, Davi O Santos ${ }^{7}$ Dalma M Banic ${ }^{3}$, Maria G Bonecini-Almeida ${ }^{7}$, Josué C Lima-Júnior ${ }^{1}$ and Joseli Oliveira-Ferreira ${ }^{1 *}$

\begin{abstract}
Background: Cytokines play an important role in human immune responses to malaria and variation in their production may influence the course of infection and determine the outcome of the disease. The differential production of cytokines has been linked to single nucleotide polymorphisms in gene promoter regions, signal sequences, and gene introns. Although some polymorphisms play significant roles in susceptibility to malaria, gene polymorphism studies in Brazil are scarce.
\end{abstract}

Methods: A population of 267 individuals from Brazilian Amazon exposed to malaria was genotyped for five single nucleotide polymorphisms (SNPS), IFNG + 874 T/A, IL10A-1082G/A, IL10A-592A/C, IL 10A-819 T/C and NOS2A-954G/C. Specific DNA fragments were amplified by polymerase chain reaction, allowing the detection of the polymorphism genotypes. The polymorphisms IL10A-592A/C and IL10A-819 T/C were estimated by a single analysis due to the complete linkage disequilibrium between the two SNPs with $D^{\prime}=0.99$. Plasma was used to measure the levels of IFN- $\gamma$ and IL-10 cytokines by Luminex and nitrogen radicals by Griess reaction.

Results: No differences were observed in genotype and allelic frequency of IFNG +874 T/A and NOS2A-954G/C between positive and negative subjects for malaria infection. Interesting, the genotype NOS2A-954C/C was not identified in the study population. Significant differences were found in IL10A-592A/C and IL10A-819 T/C genotypes distribution, carriers of IL 10A -592A/-819 T alleles (genotypes AA/TT + AC/TC) were more frequent among subjects with malaria than in negative subjects that presented a higher frequency of the variant $C$ allele $(p<0.0001)$. The presence of the allele C was associated with low producer of IL-10 and low parasitaemia. In addition, the GTA haplotypes formed from combinations of investigated polymorphisms in IL10A were significantly associated with malaria (+) and the CCA haplotype with malaria (-) groups. The IL10A-1082G/A polymorphism showed high frequency of heterozygous $A G$ genotype in the population, but it was not possible to infer any association of the polymorphism because their distribution was not in Hardy Weinberg equilibrium.

Conclusion: This study shows that the IL 10A-592A/C and IL10A-819 T/C polymorphisms were associated with malaria and decreased IL-10 levels and low parasite density suggesting that this polymorphism influence IL-10 levels and may influence in the susceptibility to clinical malaria.

Keywords: Malaria disease, Cytokines, Nitric oxide synthase, Polymorphism

\footnotetext{
* Correspondence: lila@ioc.fiocruz.br

'Laboratório de Imunoparasitologia, Instituto Oswaldo Cruz/Fiocruz, Av. Brasil

4365, Manguinhos, Rio de Janeiro, Brazil

Full list of author information is available at the end of the article
} 


\section{Background}

Malaria is an infectious disease that affects millions of people each year worldwide. The species of Plasmodium that affect humans have different pathogenic potential. However, beyond the pathogenic potential of the parasite, there are environmental factors, host genetics and parasite virulence associated to susceptibility and resistance to malaria [1,2]. The identification of host factors may increase the understanding of the interactions between the parasite and host, as well as the mechanisms involved in the pathology and immunity. In human malaria, a link between enhanced IFN- $\gamma$, TNF, IL-6, IL-10 and nitric oxide (NO) levels and severity of the disease have long been reported [3-7], although this is not a consistent finding [8-10]. In recent years, several studies have demonstrated that the presence of polymorphisms in IFN- $\gamma$, IL-10 and NO gene have been associated with susceptibility or resistance to various diseases [11-15].

The main polymorphism in the gene encoding IFN- $\gamma$ (IFNG $+874 \mathrm{~T} / \mathrm{A}$ polymorphism) is located in its first intron at position +874 and studies have reported only weak associations between IFNG SNPs and susceptibility to severe malaria [16,17]. In Brazil, there is one study showing that IFNG $+874 T / A$ polymorphism are associated with reduced levels of IFN- $\gamma$ in patients with the homozygote mutant AA genotype while carriers of the wild alleles (AT and TT) were associated with higher levels of this cytokine [18].

IL-10 cytokine has an important regulatory role and polymorphisms in the promoter region of IL10A impair the production of this cytokine $[19,20]$, and may contribute to the pathogenesis of diseases. In malaria, the role of IL-10 in regulating the inflammatory response remain conflicting since several studies suggest that enhanced IL-10 is associated with increased pathogenesis while others associate with protection [10,21-23]. The coding gene of IL-10 cytokine contains a promoter region with at least $5 \mathrm{~kb}$, which were described over 27 polymorphisms [19]. In malaria, polymorphisms in the promoter region $(I L-10 A-1082 A / G,-819 \mathrm{~T} / \mathrm{C}$ and $-592 A / C)$ were associated with reduced IL-10 plasma levels and with the development of acute anaemia in Kenyan children with P. falciparum malaria in holoendemic areas [20].

Nitric oxide synthase 2 is the critical enzyme involved in the synthesis of nitric oxide (NO), a molecule with diverse functions. There has been much speculation about the part played by nitric oxide in malaria, both as an antiparasitic agent and as a potential cause of cerebral malaria [24-27]. A report from Gabon suggests that a single nucleotide polymorphism in the inducible nitric oxide synthase $(N O S 2 A)$ promoter is associated with protection from all forms of severe malaria, including susceptibility to reinfection while other study report an association with the risk of fatal cerebral malaria [28].
Recently, an association was found between mutation of a nucleotide at position 84 in the gene of the enzyme, $N O S 2 A$ and a higher risk of cerebral malaria [29]. Moreover, associations between protection against severe malaria and polymorphic forms of the promoter region in African children have also been described [26]. However, few studies evaluate the effect of the gene polymorphism in the promoter of the NOS $2 A$ gene in NO production. Although some polymorphisms play significant roles in susceptibility to malaria, several findings are inconclusive and contradictory and studies that explore the influence of these polymorphisms in Brazil is scarce [18,30-32]. Thus, cytokine gene polymorphisms have an unquestionable role in the orchestration of the immune response, leading to different functional scenario, which in turn influence the outcome of disease establishment and evolution.

The hypothesis is that SNP polymorphisms may result in changes in recognition sites of some transcription factors that influence the levels of pro-and anti-inflammatory cytokines in malaria infection and may lead to imbalance between these molecules that could favor the host susceptibility to Plasmodium and increase the risk for clinical malaria in individuals naturally exposed to infections. Therefore, this study examined the SNPs polymorphisms that affect the expression of genes encoding IFN- $\gamma$ (-874 T/A), IL-10 (-1082A/G, -819 T/C and $-592 \mathrm{~A} / \mathrm{C})$ and iNOS (-954G/C) from a Brazilian Amazonian population living in malaria endemic area of Brazil.

\section{Methods}

\section{Subjects and methods}

The present study included 267 individuals (malaria-exposed group) from Porto Velho, Rondônia State, malaria endemic area in the southwestern Brazilian Amazon. Among these individuals 73 (27.3\%) were positive for malaria infection (malaria (+) group) and 194 (72.7\%) individuals living in the same area were negative for malaria infection (malaria (-) group). Malaria diagnosis was performed in Giemsa-stained thin and thick blood smears and parasitological evaluation was done by examination of 200 fields at $1,000 \mathrm{X}$ magnification under oilimmersion. The parasitaemia was expressed as the number of parasites/ $\mu$ l of blood in the thick blood smear. The number of parasites/ $\mu \mathrm{l}$ of blood was calculated by multiplying the number of parasites counted against 500 leucocytes, and the number of leukocytes of the subject and dividing the product by 500 . A researcher expert in malaria diagnosis examined all slides. To confirm the parasitological diagnosis, molecular analyses of all samples was performed using primers specific for genus (Plasmodium sp.) and species (Plasmodium falciparum and Plasmodium vivax). The amplification protocols were described previously by Snounou et al. [33]. Subjects were considered to have malaria if they were positive in the thick blood smear and/or 
polymerase chain reaction (PCR). Positive volunteers for $P$. vivax and/or P. falciparum at the time of blood collection were subsequently treated using the chemotherapeutic regimen recommended by the Brazilian Ministry of Health [34]. Written informed consent was obtained from all volunteers and the study was reviewed and approved by the Fundação Oswaldo Cruz Ethical Committee and the Brazilian National Ethical Committee. All volunteers were clinically evaluated and answered an epidemiological questionnaire, including data as age, gender, time of residence in endemic area, number of past infections, past and last Plasmodium species infection and time since last infection.

\section{DNA extraction and genotyping}

Genomic DNA was extracted using the kit QIAamp ${ }^{\oplus}$ DNA Blood Midi/Maxi (QIAgen, Hilden, Germany), quantified using the NanoDrop ND-1000 and stored at $-20^{\circ} \mathrm{C}$ until use. Amplification Refractory Mutation System (ARMS-PCR), first described by Newton et al. [35], analysed single nucleotide polymorphisms (SNPs) for the IFNG + 874 T/A, IL-10A-1082A/G, IL10A$819 \mathrm{~T} / \mathrm{C}$ and IL10A-592A/C, and NOS2A-954G/C polymorphism by Restriction fragment length polymorphism (RFLP) [26]. The polymorphisms IL10A$592 A / C$ and $I L 10 A-819 T / C$ were estimated by a single analysis due to the complete linkage disequilibrium between the two SNPs with D'=0.99. Amplifications were performed in a GeneAmp PCR System 9700 (Applied Biosystems, Foster City, CA) using 2.5UI for IFNG and ILIOA and 1.5UI for NOS2A of Taq DNA polymerase $(5 \mathrm{U} / \mu \mathrm{L}$, Invitrogen). Cycling PCR conditions for IFNG $+874 \mathrm{~T} / \mathrm{A}$ were $95^{\circ} \mathrm{C}$ (3 minutes), 10 cycles of $95^{\circ} \mathrm{C}$ (15 seconds), $65^{\circ} \mathrm{C}$ (50 seconds), $72^{\circ} \mathrm{C}$ (40 seconds) followed by 20 cycles of $95^{\circ} \mathrm{C}(20 \mathrm{sec}-$ onds), $55^{\circ} \mathrm{C}$ (50 seconds) and $72^{\circ} \mathrm{C}$ (50 seconds), $72^{\circ} \mathrm{C}$ (7 minutes) $4^{\circ} \mathrm{C}$ until use. Cycling PCR conditions for IL $10 \mathrm{~A}$ polymorphisms were $95^{\circ} \mathrm{C}$ ( 1 minute), 10 cycles of $95^{\circ} \mathrm{C}$ ( 15 seconds), $65^{\circ} \mathrm{C}$ (50 seconds), $72^{\circ} \mathrm{C}$ (40 seconds) followed by 20 cycles of $95^{\circ} \mathrm{C}$ (20 seconds), $59^{\circ} \mathrm{C}$ (50 seconds) and $72^{\circ} \mathrm{C}$ (50 seconds), $4^{\circ} \mathrm{C}$ until use. Cycling PCR conditions for NOS2A-954G/C were $95^{\circ} \mathrm{C}$ (3 minutes), 30 cycles of $94^{\circ} \mathrm{C}$ (10 seconds), $60^{\circ} \mathrm{C}$ (30 seconds), $72^{\circ} \mathrm{C}$ (30 seconds); $72^{\circ} \mathrm{C}$ (7 minutes) and $4^{\circ} \mathrm{C}$ until use. The NOS2A-954G/C amplified product was subsequently digested with $\mathrm{BSAI}$ restriction enzyme in the following condition: $50^{\circ} \mathrm{C}$ (60 minutes), $65^{\circ} \mathrm{C}$ (20 minutes) and $4^{\circ} \mathrm{C}$ until use. All amplified products were evaluated by electrophoresis on a $1.5 \%$ (IFNG and IL1OA) and $2.5 \%(N O S 2 A)$ agarose gel containing ethidium bromide $(0.5 \mu \mathrm{g} / \mathrm{mL})$. After electrophoresis, the fragments were displayed and the images were photographed on a transilluminator Multi Doc-It ${ }^{\mathrm{TM}}$ Digital Imaging System (UVP, Upland, CA) (Figure 1).

\section{Analysis of plasma cytokines concentration}

Levels of IFN- $\gamma$ and IL-10 were detected in plasma samples by a multiplex assay (Bio-Plex assay, Bio-Rad Laboratories, Hercules, CA, USA) according to the manufacturer's instructions using the Luminex system (Luminex Corporation, Austin, TX, USA) and analysed with a Bio-Plex suspension array system (Bio-Rad Laboratories). Fluorescence intensity was transformed into cytokine concentration using the Bio-Plex manager software (version 3.0). A minimum of 100 beads per region were analysed. A curve fit was applied to each standard curve according to the manufacturer's manual and sample concentrations were interpolated from the standard curves. The limit of detection was $0.79 \mathrm{pg} / \mathrm{mL}$ for IFN- $\gamma$ and $2 \mathrm{pg} / \mathrm{mL}$ for IL-10.

\section{Griess microassay detection of nitrite and nitrate}

A modified Griess reaction was used to detect nitrite and nitrate [36,37]. The NO levels in samples were indirectly measured after first converting nitrates to nitrites with a nitrate reductase treatment (Aspergillus species NAD [P] H, Sigma, UK) and NADPH $\beta$-nicotinamide adenine dinucleotide phosphate (Sigma Diagnostics, St. Louis, USA). Griess reagent [5\% phosphoric acid, $1 \%$ sulphanilic acid and $0.1 \% \mathrm{~N}$-(1-naphthyl-1)-ethylendiaminedihydrochloride, all from Sigma, UK, dissolved in $100 \mathrm{~mL}$ deionized water] was added and proteins were subsequently precipitated by trichloroacetic acid $(\mathrm{BDH}$, England). The tube contents were mixed and centrifuged (Eppendorf centrifuge $5415 \mathrm{C}$, Germany); two samples of each supernatant were transferred to a flat-bottomed microplate and their absorbances were read at $520 \mathrm{~nm}$ using a microplate reader (SpectraMax, Molecular Devices Inc). NO values were calculated from standard calibration plots.

\section{Statistical analysis}

Epidemiological and experimental data were stored in the Epi- Info 3.5.1 (CDC, Atlanta, USA). Allele and genotype frequencies were estimated by gene counting and differences between groups by $x^{2}$ test. The risk of malaria associated with polymorphisms was estimated using odds ratios (OR) and confidence interval of 95\% (CI) with and without adjustment by age, gender, length of residence in an endemic area and number of previous episodes of malaria. Differences between malaria (+) and malaria (-) groups were estimated by Mann-Whitney and t tests. Relationships between epidemiological factors and cytokine levels were assessed by Spearman's correlation The Hardy-Weinberg equilibrium (HWE) was assessed by $\chi^{2}$ test and Fisher Person. The genetic analyses correspond to codominant logistic regression model (homozygous major allele VS. Heterozygote + homozygote secondary). The linkage disequilibrium 


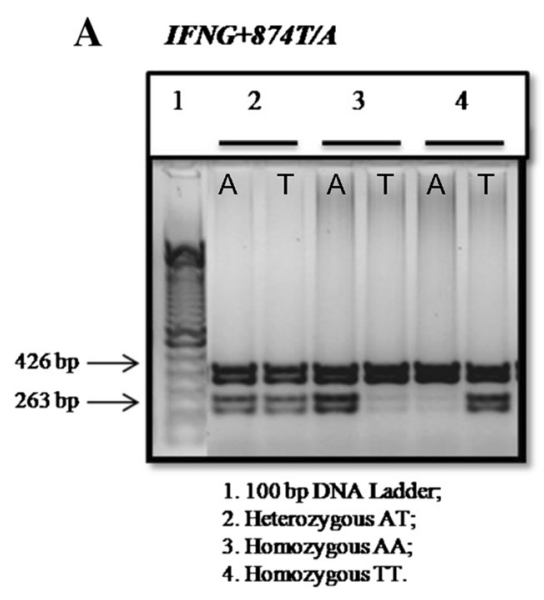

\section{IL10A-592A/Cand IL10A-819T/C}

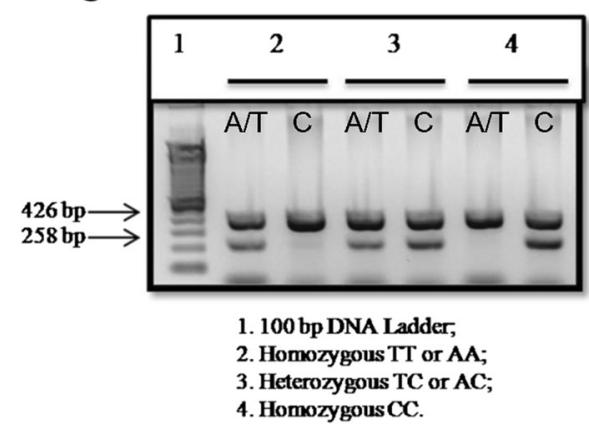

B NOS2A-954G/C

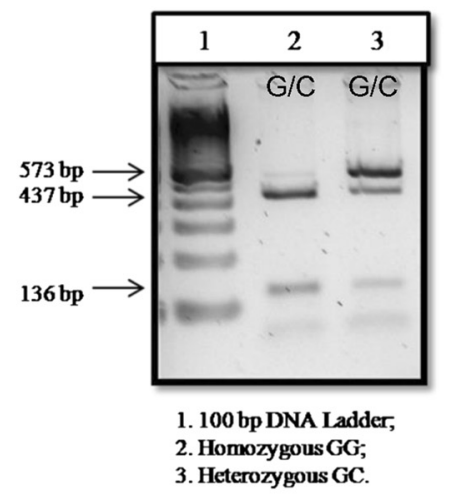

D H10A-1082G/A

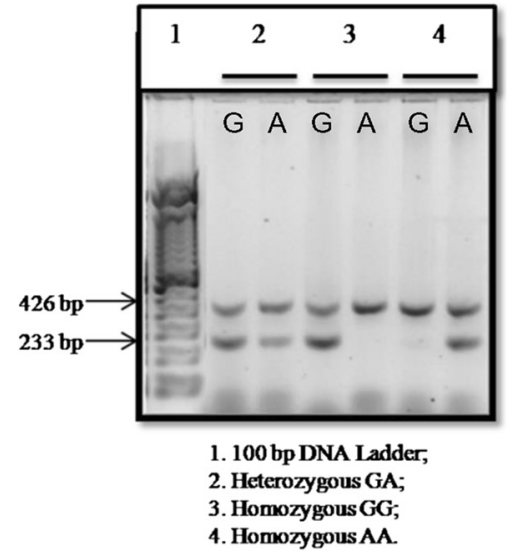

Figure 1 Visualization of amplified fragments on agarose gel. 426 bp fragment: band corresponding to internal control using a pair of primers designed from the nucleotide sequence of human growth hormone; A. 263 bp fragment: specific bands for T and A alleles of IFNG + 874 T/A polymorphism; B. 437 and 136 bp fragments: specific bands for $\mathrm{G}$ allele of NOS2A-954G/C polymorphism; 573 bp fragment: specific band for C allele of NOS2A-954G/C polymorphism; C. 258 bp fragment: specific bands for A/T and C alleles of IL10A-592A/C and IL10A-819 T/C polymorphisms were estimated by a single analysis due to the complete linkage disequilibrium of the two SNPs $\left(D^{\prime}=0.99\right)$, D. 233 bp fragment: specific bands for $\mathrm{G}$ and $\mathrm{A}$ alleles of IL10A-1082G/A polymorphism.

was calculated by D statistic and frequency haplotypes by calculating the maximum likelihood estimator via the EM algorithm (Expectation Maximization) using two-step, step "E" (Hope) and step "M" (max) until convergence is achieved. Statistical analyses were performed using Graph Pad Prism software and SNPStats 5.0 (San Diego, California, USA). Statistically significant values of $\mathrm{p}<0.05$ was considered.

\section{Results}

Description of the studied population

The epidemiological surveillance shows that a significant proportion (93.16\%) of the inhabitants reported a prior infection with $P$. vivax and/or P. falciparum indicating that the majority of the individuals were exposed to malaria parasite throughout the years.
Among studied participants, 55.4\% were male and the mean age was $30.99 \pm 14.37$ years. Comparing both groups, a higher frequency of males was observed in malaria (+) group. However, no statistical differences were identified regarding age, the length of residence in an endemic area and number of previous episodes of malaria between groups. Yet, the time since last infection was higher in malaria (-) group (Table 1$)$. In malaria (+) group, 53 (72.6\%) individuals were infected with P. vivax and 20 (27.4\%) with P. falciparum, consistent with Plasmodium species distribution reported by the Brazilian Ministry of Health [34]. No cases of severe malaria were seen and fever and headache were the main reported symptoms in all patients. Parasitaemia and symptoms were similar in patients infected with both plasmodial species (Table 2). 
Table 1 Characteristics of the study participants

\begin{tabular}{|c|c|c|c|c|}
\hline & \multicolumn{2}{|c|}{ Malaria diagnosis } & \multirow[b]{2}{*}{ Total $N=267$} & \multirow[b]{2}{*}{${ }^{*} P$ value } \\
\hline & Positive $\mathrm{N}=73$ & Negative $N=194$ & & \\
\hline \multicolumn{5}{|l|}{ Gender n (\%) } \\
\hline q & $21(28.8 \%)$ & $98(50.5 \%)$ & $119(44.6 \%)$ & 0.001 \\
\hline$\widehat{\theta}$ & $52(71.2 \%)$ & $96(49.5 \%)$ & $148(55.4 \%)$ & 0.001 \\
\hline Age & $28(9-54)$ & $30(4-71)$ & $29(4-71)$ & 0.531 \\
\hline TREA (years) & $25(1-33)$ & $24(2-32)$ & $24(1-63)$ & 0.079 \\
\hline NPE & $5(0-50)$ & $5(0-99)$ & $5(0-99)$ & 0.072 \\
\hline TLI (Months) & $6(0-360)$ & $12.5(0-420)$ & $12(0-420)$ & 0.016 \\
\hline
\end{tabular}

Data expressed as $\mathrm{n}$ (\%) of patients; Time of Residence in years TREA; Number of Previous Episodes NPE; Time since Last Infection TLI; Age, TREA, NPE and TLI expressed as median (minimum-maximum); *Statistical significance determined by Mann Whitney $\mathrm{U}$ and Chi-square tests.

\section{IFN- $\gamma$, IL-10 and NO plasma levels}

The means levels of IFN- $\gamma(66.71 \mathrm{pg} / \mathrm{mL}[2.00-3629.00])$ and IL-10 (795.34 pg/mL [1.15-16782.93]), were higher in plasma of malaria $(+)$ when compared with malaria (-) group (IFN- $\gamma$ 19.44 pg/mL [1.46-3919.00] and IL-10 $1.29 \mathrm{pg} / \mathrm{mL}$ [0.40-989.00]). The levels of NO did not differ significantly when the groups were compared (malaria (+) 25.49 $\mu \mathrm{M}$ [11.65-75.85] and malaria (-) $35.41 \mu \mathrm{M}$ [0.52-110.95]) (Figure 2A). No differences were observed in the levels of IFN- $\gamma(58.96 \mathrm{pg} / \mathrm{mL}$ [23629]), IL-10 (627.39 pg/mL [1.15-16782.9]) and NO levels $(23.98 \mu \mathrm{M}$ [11.65-75.85]) in P. vivax $(\mathrm{n}=53)$ infected individuals when compared with the levels of IFN- $\gamma$ (98.40 pg/mL [2.00-548.15]), IL-10 (1149.88 pg/ $\mathrm{mL}$ [3.02-6540.45]) and NO levels (28.49 $\mu \mathrm{M}$ [12.9940.31]) in $P$. falciparum $(\mathrm{n}=20)$ infected individuals (Figure 2B). Although both IL-10 and IFN- $\gamma$ were higher in malaria $(+)$ group, Figure 3 shows that the correlation between cytokine levels and parasite density were only observed for IL-10 (rho $=0.58 \mathrm{p}<0.0001$ ).

Alleles, genotypes and haplotypes frequencies obtained for IFNG, IL10A and NOS2A genes

The SNPs investigated, their location in the gene, the genotype and allele frequencies observed in malaria (+) and malaria (-) groups are presented in Table 3. The groups fell within Hardy-Weinberg equilibrium with non-significant values by $x^{2}$ test for the genotype observed and expected for all polymorphism except for the IL10A-1082G/A $\left(\mathrm{X}^{2}=41.76, \mathrm{p}<0.0001, \mathrm{\chi}^{2}=32.33, \mathrm{p}<\right.$ $0.0001)$. The IFNG $+874 T / A$ genotype and allelic frequencies did not differ between malaria $(+)$ and malaria (-) groups. In both groups, the most frequent genotype for IFNG $+874 T / A$ was the homozygous mutant AA followed by AT heterozygous and homozygous wild $\mathrm{T}$ allele. The variant allele $+874 \mathrm{~A}$ was more frequent than wild T. In contrast, the distribution of the IL10A-1082G/ $A$ genotype differed between malaria (+) and malaria (-) groups while allelic frequencies were similar in both groups. Although the heterozygous AG genotype were more frequent in both groups, this genotype was significantly higher in malaria $(+)(\mathrm{p}<0.001)$. The polymorphisms IL10A-592A/C and IL10A-819 T/C were estimated by a single analysis due to the complete linkage disequilibrium between the two SNPs with $\mathrm{D}^{\prime}=0.99$. The A allele of $I L 10 A-592 A / C$ was always linked with the T allele of and IL10A-819 T/C and C with C. IL10A-592A/ $\mathrm{A}$ and IL10A-819 T/T genotypes were more frequently observed in malaria $(+)$ compared with malaria $(-)$ group $(\mathrm{p}<0.001)$, even when adjusted by sex and age. Similarly

Table 2 Malaria symptoms and parasitaemia according to plasmodial species

\begin{tabular}{|c|c|c|c|c|}
\hline \multicolumn{4}{|l|}{ Plasmodial species $(n=73)$} & \multirow[b]{2}{*}{${ }^{*} P$ value } \\
\hline & P. vivax & P. falciparum & Total & \\
\hline Number of infected $\mathrm{n}(\%)$ & $53(72.6 \%)$ & $20(27.4 \%)$ & 73 & $<0.0001$ \\
\hline Parasitaemia & $2286(50-17933)$ & $1214(52-12623)$ & $2097(50-17933)$ & 0.334 \\
\hline Days since the onset of symptoms & $2(1-5)$ & $3(1-10)$ & $3(1-10)$ & \\
\hline Fever & $38(71.7 \%)$ & $15(75 \%)$ & $53(72.6 \%)$ & 0.779 \\
\hline Headache & $40(75.5 \%)$ & $15(75 \%)$ & $55(75.3 \%)$ & 0.968 \\
\hline Chill & $32(60.4 \%)$ & $13(65 \%)$ & $45(61.6 \%)$ & 0.719 \\
\hline Myalgia & $31(58.5 \%)$ & $14(70 \%)$ & $45(61.6 \%)$ & 0.368 \\
\hline Nausea & $20(37.7 \%)$ & $11(55 \%)$ & $31(42.5 \%)$ & 0.184 \\
\hline
\end{tabular}

Data expressed as $\mathrm{n}$ (\%) of patients; Parasitaemia (number of parasites/ $\mathrm{L}$ of blood) and onset of symptoms expressed as median (minimum-maximum); *Statistical significance determined by Mann Whitney $U$ and Chi-square tests. 

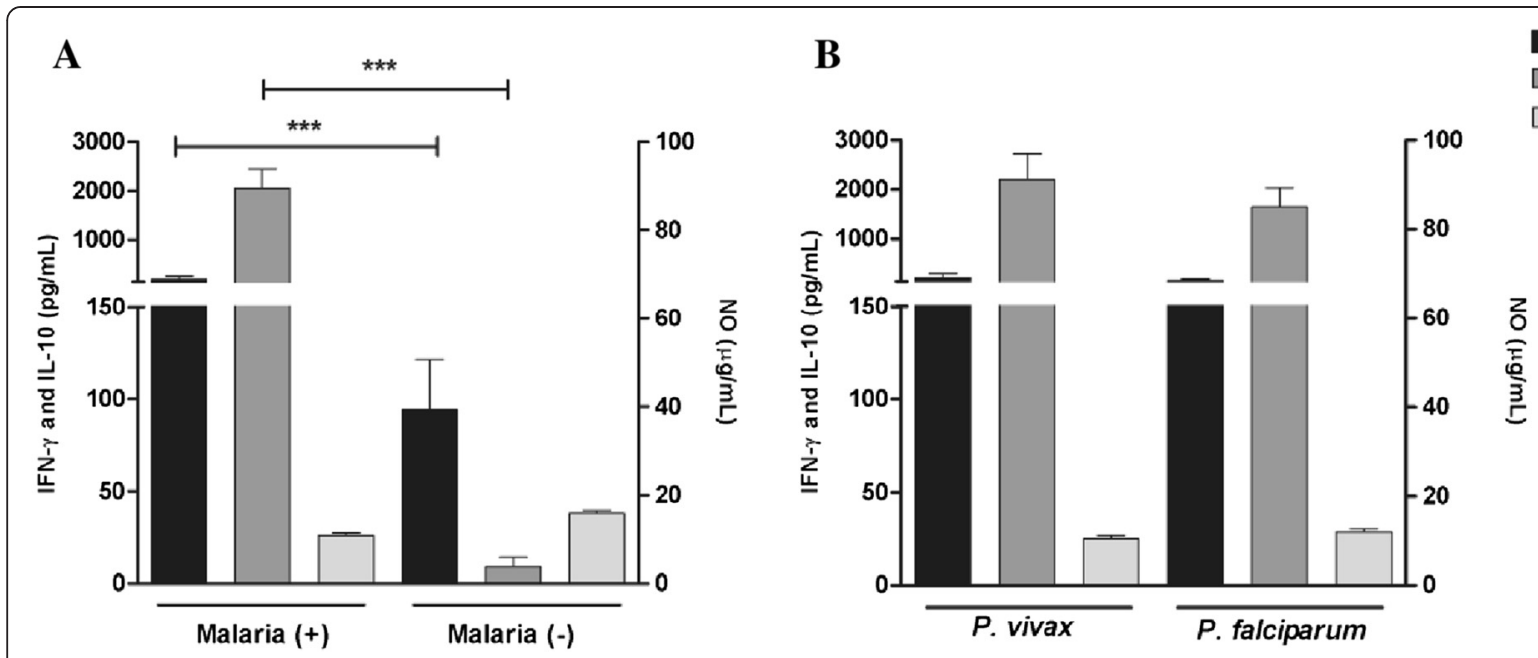

Figure 2 Levels of IFN- $\gamma(\mathrm{pg} / \mathrm{mL}), \mathrm{IL}-10(\mathrm{pg} / \mathrm{mL})$ and NO $(\mu \mathrm{g} / \mathrm{mL})$ were compared in Malaria $(+)$ and Malaria $(-)$ groups and according to the plasmodial species. Levels of IFN-y, IL-10 and NO in: A. Malaria (+) and Malaria (-) groups and $\mathbf{B}$. Plasmodium vivax and Plasmodium falciparum infections. Data are expressed as means levels (means \pm SEM) on a logarithmic scale; Statistical significance determined by Mann Whitney $U$ test; ${ }^{* * *} P$ value $<0.0001$.

the wild alleles $\mathrm{A} / \mathrm{T}$ were more frequent in malaria $(+)$ than malaria $(-)$ groups $(\mathrm{OR}=0.45 ; \mathrm{p}<0.0001)$. Stratification of the individuals into haplotypic groups based on the tree promoter IL10A polymorphisms at positions $-592 /$ 819/-1082 yielded the following haplotypes distribution: Hap1 (CCG), Hap2 (ATA), Hap3 (CCA) and Hap4 (ATG) with frequencies that varied from $7 \%$ to $38 \%$ in both malaria $(+)$ and malaria (-) groups. As shown in Table 4, Hap1 and Hap2 were the most frequent haplotypes with similar distribution in the groups. The distribution of the haplotypes Hap3 and Hap4 were significantly different between malaria $(+)$ and malaria $(-)$ groups, while the Hap1 and Hap2 were similar between the groups. The Hap3 haplotype was more frequent in malaria (-) while the Hap4 were more frequent in malaria $(+)$ group. The distribution of NOS2A-954G/C genotype in the population showed that the homozygous GG was more frequent in both groups and the genotype NOS2A-954C/C was not identified in the study population. The $\mathrm{G}$ allele was also the most frequent allele with similar distribution in both groups.

\section{Association between IFNG, IL-10 and NOS2A genotypes and their products}

No association was observed between IFNG-874 T/A, NOS2A-954G/C and IL10A-1082G/A SNPs and the levels of their products in plasma (Figure $4 \mathrm{~A}-\mathrm{C}$ ). However, individuals with $I L 10 A-592 A / C$ and $I L 10 A-819$ T/C genotypes were strongly associated with the plasma levels of IL- 10 . The IL-10 levels were lower in subjects who carried the
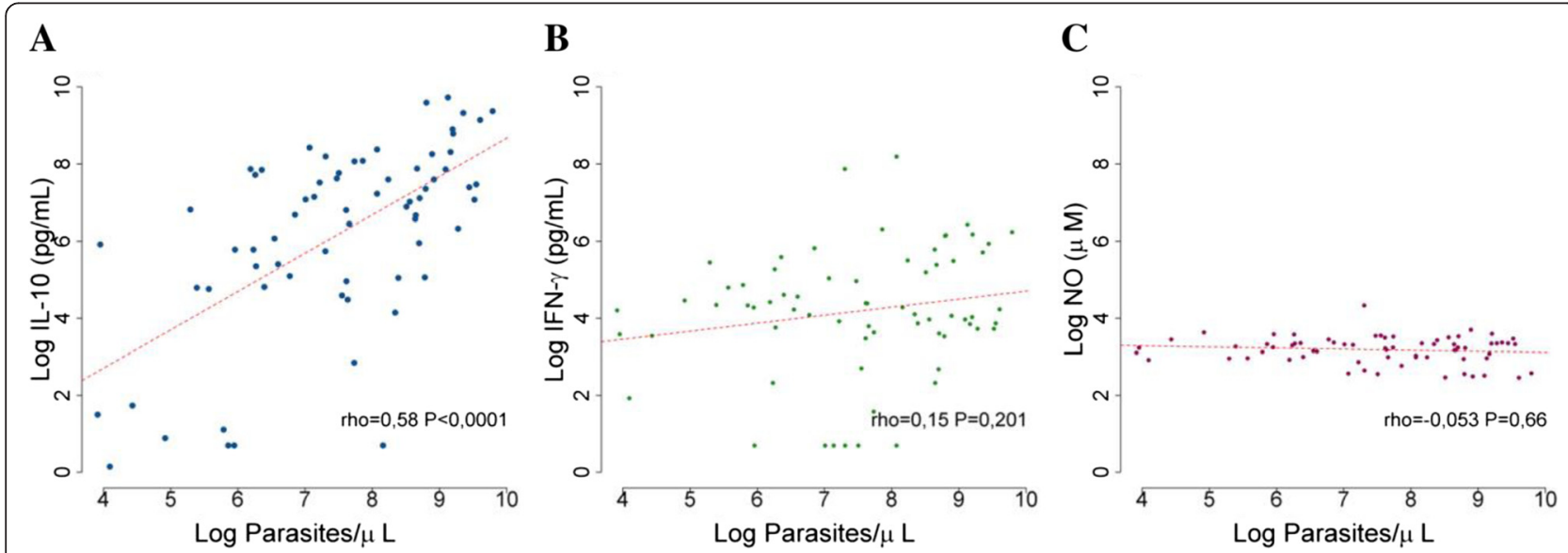

Figure $\mathbf{3}$ Correlation of cytokines and nitrogen radicals levels with parasite density in malaria patients. A. IL-10 levels, B. IFN-ץ levels and C. NO levels. High titers of IL-10 serum levels were correlated with parasitaemia by Spearman correlation $(r h o=0.58, p<0.0001)$. 
Table 3 Genotypic and allelic frequencies of single nucleotide polymorphisms in malaria (+) and malaria (-) groups

\begin{tabular}{|c|c|c|c|c|c|}
\hline Studied polymorphisms & & $\begin{array}{l}\text { Malaria }(+) \\
(n=73)\end{array}$ & $\begin{array}{l}\text { Malaria }(-) \\
(n=194)\end{array}$ & OR $(95 \% \mathrm{Cl})$ & ${ }^{*} P$ value \\
\hline \multicolumn{6}{|l|}{ IFNG + $874 T / A$} \\
\hline \multirow[t]{3}{*}{ Genotypes } & $\mathrm{A} / \mathrm{A}$ & $38(52 \%)$ & $101(52.1 \%)$ & 1.00 & 0.92 \\
\hline & $A / T$ & $30(41.1 \%)$ & 77 (39.7\%) & $0.97(0.55-1.70)$ & \\
\hline & $\mathrm{T} / \mathrm{T}$ & $5(6.8 \%)$ & $16(8.2 \%)$ & $1.20(0.41-3.51)$ & \\
\hline \multirow[t]{2}{*}{ Alleles } & A & $106(73 \%)$ & $279(72 \%)$ & $0.16(0.68-1.58)$ & 0.87 \\
\hline & $\mathrm{T}$ & $40(27 \%)$ & $109(28 \%)$ & & \\
\hline \multicolumn{6}{|l|}{$N O S 2 A-952 G / C$} \\
\hline \multirow[t]{3}{*}{ Genotypes } & $\mathrm{G} / \mathrm{G}$ & 70 (95.9\%) & $183(94.3 \%)$ & 1.00 & 0.6 \\
\hline & $\mathrm{G} / \mathrm{C}$ & $3(4.1 \%)$ & $11(5.7 \%)$ & $1.40(0.38-5.18)$ & \\
\hline & $\mathrm{C} / \mathrm{C}$ & - & - & - & \\
\hline \multirow[t]{2}{*}{ Alleles } & G & 143 (98\%) & 377 (97\%) & $1.39(0.38-5.05)$ & 0.6 \\
\hline & C & $3(2 \%)$ & $11(3 \%)$ & & \\
\hline \multicolumn{6}{|l|}{ IL10A-1082G/A } \\
\hline \multirow[t]{3}{*}{ Genotypes } & $\mathrm{A} / \mathrm{A}$ & $3(4.1 \%)$ & $40(20.6 \%)$ & 1.00 & 0.0009 \\
\hline & $\mathrm{G} / \mathrm{A}$ & $64(87.7 \%)$ & $135(69.6 \%)$ & $0.16(0.05-0.53)$ & \\
\hline & $\mathrm{G} / \mathrm{G}$ & $6(8.2 \%)$ & $19(9.8 \%)$ & $0.24(0.05-1.05)$ & \\
\hline \multirow[t]{2}{*}{ Alleles } & A & $70(48 \%)$ & $215(62 \%)$ & $0.74(0.50-1.08)$ & 0.12 \\
\hline & G & $76(52 \%)$ & $173(45 \%)$ & & \\
\hline \multicolumn{6}{|c|}{ IL10A-592A/C and IL10A-819 T/C } \\
\hline \multirow[t]{3}{*}{ Genotypes } & $\mathrm{C} / \mathrm{C}$ & $13(17.8 \%)$ & $70(36.1 \%)$ & 1.00 & 0.0002 \\
\hline & $\mathrm{A} / \mathrm{C} ; \mathrm{T} / \mathrm{C}$ & $36(49.3 \%)$ & $100(51.1 \%)$ & $0.52(0.26-1.04)$ & \\
\hline & $\mathrm{A} / \mathrm{A} ; \mathrm{T} / \mathrm{T}$ & $24(32.9 \%)$ & $24(12.4 \%)$ & $0.19(0.08-0.42)$ & \\
\hline \multirow[t]{2}{*}{ Alleles } & C & $62(42 \%)$ & $240(62 \%)$ & $0.45(0.30-0.67)$ & 0.0001 \\
\hline & $\mathrm{A} / \mathrm{T}$ & $84(58 \%)$ & $148(38 \%)$ & & \\
\hline
\end{tabular}

IFNG + 874 T/A, NOS2A-954G/C, IL10A-1082G/A, IL10A-592A/C and IL10A-819 T/C polymorphisms: Alleles, $\mathrm{n}$ (\%); Genotypes $\mathrm{n}$ (\%); OR (95\% CI), calculating odds ratios with confidence interval $(\mathrm{Cl})$ of $95 \%$; ${ }^{*}$ Analysis by $X^{2}$ test using the Fisher model codominance.

homozygous variant IL10A-592CC and -819CC (1.19 pg/ $\mathrm{mL}$ [0.4-2241]) compared to subjects with IL10A-592 AC and -819TC (2.3 pg/mL [0.4-11233]) and wild homozygous individuals with IL10A-592AA/-819TT $(18.4 \mathrm{pg} / \mathrm{mL}$ [0.4-16782] $)(\mathrm{p}<0.0001)$. Wild homozygous individuals with IL10A-592AA/-819TT presented IL-10 levels three times higher than individuals carrying variant allele $C$, indicating an association between the AA/TT genotypes with clinical malaria risk (Figure 4D).
Polymorphisms and parasitaemia levels association

The parasite densities among the different genotypes and carried alleles were evaluated and the parasitaemia was not influenced by the IFNG $+874 T / A, N O S 2 A-$ 954G/C and $I L 10 A-1082 G / A$ polymorphisms (Figure 5). Among the IFNG $+874 T / A$ genotypes, the median parasitaemia was $37.85(50-17933)$ parasites/ $\mu \mathrm{L}$ for heterozygous genotype AT, $11.72(199-6518)$ parasites/ $\mu \mathrm{L}$ for homozygous wild TT and 1861 (52-11546) parasites/

Table 4 Haplotypes frequencies of IL10A in malaria (+) and malaria (-) groups

\begin{tabular}{|c|c|c|c|c|c|c|c|}
\hline \multicolumn{8}{|c|}{ Haplotypes frequencies } \\
\hline & -592 & -819 & -1082 & Malaria (+) & Malaria (-) & OR $(95 \% \mathrm{Cl})$ & ${ }^{*} P$ value \\
\hline Hap 1 & C & C & G & 0.3257 & 0.37 & 1.00 & - \\
\hline Hap2 & A & $\mathrm{T}$ & A & 0.3805 & 0.3056 & $0.85(0.40-1.77)$ & 0.66 \\
\hline Hap3 & C & C & A & 0.0989 & 0.2486 & $0.36(0.14-0.93)$ & 0.036 \\
\hline Hap4 & A & $\mathrm{T}$ & G & 0.1948 & 0.0759 & $3.83(1.40-10.45)$ & 0.009 \\
\hline
\end{tabular}

-592/-819/-1082: Positions of polymorphisms in the promoter region of the interleukin 10 gene; OR ( $95 \% \mathrm{Cl}$ ), calculating odds ratios with confidence interval (Cl) of $95 \%$; *Analysis by calculating EMV via EM algorithm using a logistic regression model. 

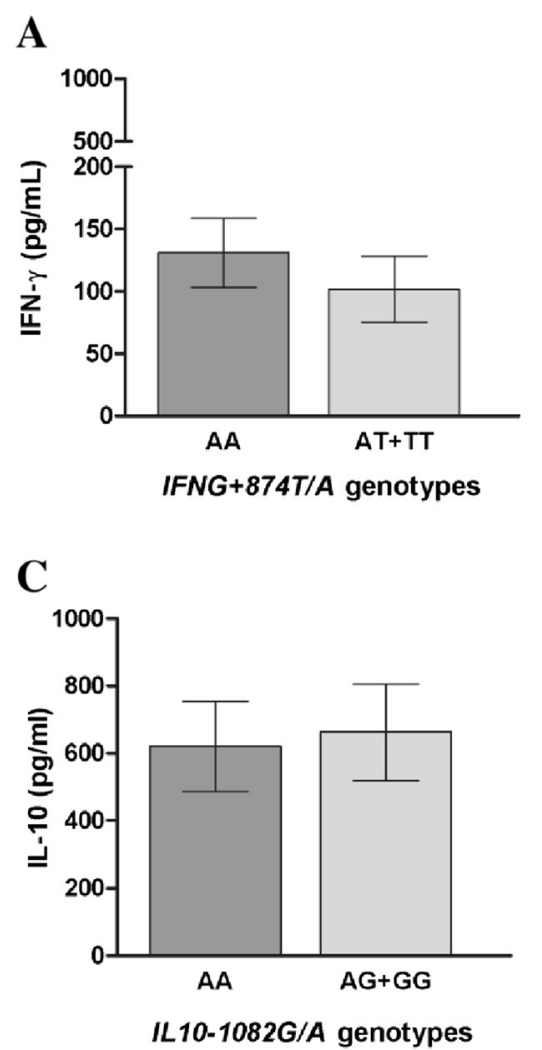

B

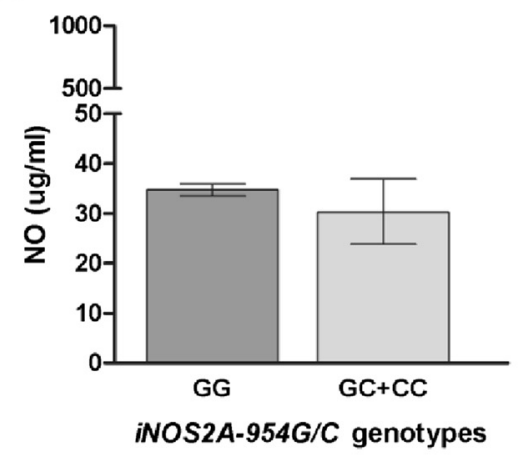

D

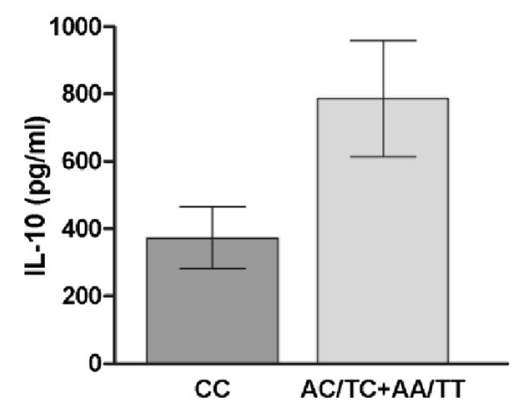

IL10-592A/C and IL10-819T/C genotypes

Figure 4 IFN- - , IL-10 and nitrogen radicals levels by different alleles carried of polymorphisms. A. IFN- $\gamma$ levels in IFNG-874 T/A genotypes, B. NO levels in NOS2A-954G/C genotypes, C. IL-10 levels in IL10A-1082G/A genotypes, D. IL-10 levels in IL10A-592A/C and IL10A-819 T/C genotypes. Data are expressed as means levels (means \pm SEM) on a logarithmic scale; Statistical significance determined by Mann Whitney $U$ test; **P value $<0.001$.

$\mu \mathrm{L}$ for homozygous variant AA (Figure 5A). Among the NOS2A-954G/C polymorphism, the homozygous CC variant was not detected and the median parasite density was 1758 (524-8888) parasites/ $\mu \mathrm{L}$ for GC genotype and 2120 (50-17933) parasites/ $\mu \mathrm{L}$ for homozygous GG (Figure 5B). The IL10A-1082G/A genotypes, the median parasitaemia was 1485 (510-3210) parasites $/ \mu \mathrm{L}$ for the homozygous AA variant, $2200(50-17933)$ parasites $/ \mu \mathrm{L}$ for the heterozygous AG and 1906 (84-6000) parasites/ $\mu \mathrm{L}$ for homozygous GG wild (Figure 5C). The IL10A592AA and IL10A-819TT genotypes presented 2440 (52-17933) parasites/ $\mu \mathrm{L}$ while the heterozygous IL10A$592 \mathrm{AC}$ and IL10A-819TC presented 2280 (84-14891) parasites $/ \mu \mathrm{L}$ (Figure 5D). These genotypes (carriers of the wild type) presented higher parasites densities when compared with the homozygous variant IL10A-592CC and IL10A-819CC 1108 (50-6000) parasites/ $\mu \mathrm{L}$.

\section{Discussion}

Human populations display differences in susceptibility to many diseases and the basis for this differential susceptibility is, at least in part, genetically determined [38,39]. Significant associations between cytokine polymorphism and diseases support that cytokine gene polymorphisms have an unquestionable role in the orchestration of the immune response, leading to different functional scenario, which in turn influence the outcome of disease establishment and evolution $[38,40]$. Thus, the present study aimed at exploring cytokine and $\mathrm{NO}$ polymorphism in populations naturally exposed to malaria, residents in Rondônia State, southwestern of Brazilian Amazon in order to establish the possible implications of these polymorphisms in malaria infection as well as analysing whether the alleles and genotypes are associated with their expression. In recent years, evaluation of SNPs have been considered a common approach for testing human genetic variation [37].

The SNPs investigated include IFNG $(-874 \mathrm{~T} / \mathrm{A})$, IL10A $(-1082 \mathrm{~A} / \mathrm{G},-819 \mathrm{~T} / \mathrm{C}$ and $-592 \mathrm{~A} / \mathrm{C})$ and NOS2A $(-954 \mathrm{G} / \mathrm{C})$. Firstly, genotypic and allelic frequencies of the IFNG $+874 T / A$ polymorphism showed that the variant allele $\mathrm{A}$ was more common in the population as well as the corresponding homozygous AA genotype. The frequencies found in the study population, are in agreement with previous studies conducted in other Brazilian [18] and Colombian Amazon regions populations 


\section{A}

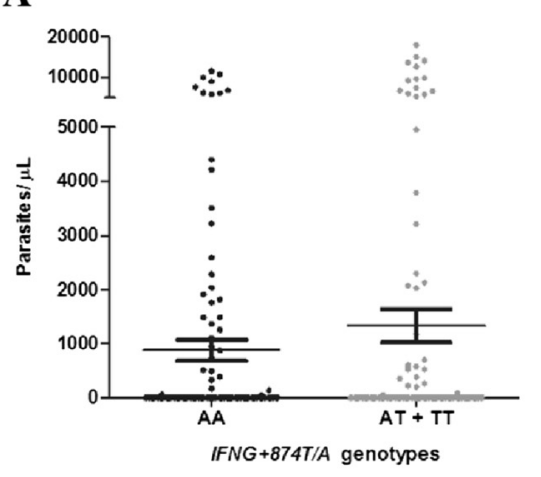

C

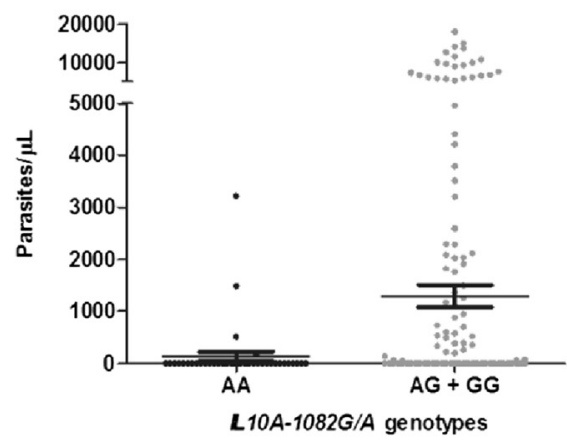

B

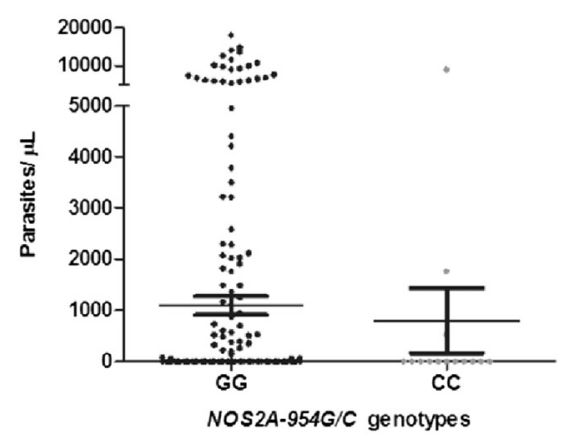

D

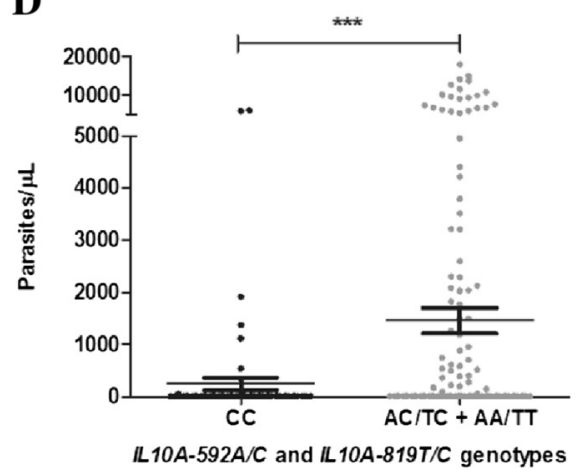

Figure 5 Parasite density and allelic carried frequencies of polymorphisms. Parasitaemia levels in: A. IFNG-874 T/A genotypes, B. NOS2A-954G/C genotypes, C. IL10A-1082G/A genotypes, D. IL10A-592A/C and IL10A-819 T/C genotypes. Data are expressed as means levels (means \pm SEM) on a logarithmic scale; Statistical significance determined by Mann Whitney $U$ test; ${ }^{* *} P$ value $<0.001$.

[41]. However, studies conducted in Brazil in nonAmazonian areas the homozygous AA genotype was predominant in patients with tuberculosis, while the control group of individuals from the same region had the heterozygous genotype predominantly AT, demonstrating a heterogeneous composition in different geographic region of Brazil, beyond the significant association of this polymorphism with tuberculosis $[11,13]$.

In the study population, no difference in the allelic and genotypic distribution of IFNG +874 T/A polymorphism was observed between malaria $(+)$ or malaria $(-)$ groups. Few studies find associations between severe malaria and IFNG $+874 T / A$ polymorphism and these associations were weak and not significant after correction for multiple comparisons $[42,43]$. In malariainfected individuals, the relationships between IFNG + 874 $T / A$ polymorphism and IFN- $\gamma$ serum levels were not observed in this study, although high levels of IFN- $\gamma$ were detected in malaria (+) subjects. The results of this study were different from Medina et al. that reported in a population of an endemic area in Brazil, a concentration of IFN- $\gamma$ significantly lower in the serum of patients with AA individuals compared with $\mathrm{T}$ wild allele carriers [18]. The possibility that there exist functional IFNG polymorphisms that were not effectively tagged by this marker cannot be excluded, and further studies of the locus are warranted.

In the $I L 10 A$ gene, investigation of three polymorphisms at positions $-592,-819$ and 1082 of the promoter region, were associated with the actual production of IL-10, which has an important role in the immune response to malaria infection $[21,44,45]$. The IL1OA gene polymorphism at position -1082 , have been associated with decreased production of IL-10 and clinical and severe malaria [7,20,46]. However, no association was found between this polymorphism and the levels of IL-10 nor with the occurrence of the disease and this polymorphism were not in HardyWeinberg equilibrium in the studied population, demonstrating the need for a representative sample to verify a possible association of this polymorphism with IL-10 levels in malaria patients. In the studied population the heterozygous GA was the most frequent genotype in both malaria (+) and malaria (-) groups. Medina et al. [18] reported the homozygous AA variant as the most common genotype in the population of Belem (Para state) but showed weak association between IL-10 concentration and parasite density [18], while a study in the Amazonas state revealed an association between $I L-10 A-1082 \mathrm{G} / \mathrm{A}$ polymorphism with reduced risk to clinical malaria [32]. Some studies that have addressed this polymorphism 
showed variation in the allele and genotype distribution according to ethnicity $[18,20,47]$. In the case of the IL1OA$592 \mathrm{~A} / \mathrm{C}$ and $-819 \mathrm{~T} / \mathrm{C}$ polymorphisms, it was observed association with IL-10 production and parasite density. Interestingly, a high prevalence of the $\mathrm{C}$ alleles and homozygous variant $-592 \mathrm{CC} /-819 \mathrm{CC}$ was found in malaria (-) group while a higher prevalence of wild alleles $\mathrm{A} / \mathrm{T}$ and homozygous genotype -592AA/-819TT in malaria (+), suggesting that the $\mathrm{C}$ allele were at lower risk to have malaria due to its prevalence in the malaria (-) group. The homozygous genotype $-592 \mathrm{CC} /-819 \mathrm{CC}$ was associated with a reduced IL-10 levels and low parasite density compared to other genotypes. Indeed, carriers of the $\mathrm{C}$ allele variant were low producers of IL-10 and presented low parasite density while carriers of $\mathrm{A} / \mathrm{T}$ wild alleles were high producer of IL-10 and presented high parasite density. The presence of the CC genotype may create site for enhanced binding of repressor that favor reduced IL-10 production.

Considering the haplotypes of ILIOA at positions $-592 /-819 /-1082$, no association was observed between the most frequent haplotypes CCG and ATA with the risk of having malaria. However, carriers of the less frequent haplotype GTA were more prevalent in malaria (+) group while carriers of the ACC haplotype were more prevalent in malaria (-) group. The haplotype analysis of these three polymorphism in the Amazonas state, the GCT allelic combination were associated with low risk of any form of malaria, this haplotype was not present in the study population in Rondônia State neither in Para State, both Amazonian endemic areas [31]. The differences in haplotypes distribution in the same region are consistent with the heterogeneous genetic profile of Brazilian population [48]. Study in Kenia reported relationship between common Africa IL10A promoter variants and protection against severe malarial anaemia and increased production of IL-10 [20]. However, other studies have shown no evidence of association between the polymorphisms in the IL-10 gene and malaria severity [18]. A study in Gambia showed an association between the haplotype of five SNPs $(+4949 \mathrm{G}$, +919C, $-627 \mathrm{G},-1117 \mathrm{C},-3585 \mathrm{~T}$ ), not evaluated in this study, and resistance to cerebral malaria and severe anaemia [49]. In Brazil, this is the first report that investigate the frequency of the promoter region haplotypes in IL10A gene associated with malaria infection, IL-10 levels and parasite density. Similar IL10A haplotypes distribution were reported in a population from the State of Para another malaria endemic area in Brazil. However, the authors did not evaluate the IL-10 levels and did not find any influence of these haplotypes in susceptibility to malaria [31].

Finally, it was not found association between NOS2A954G/C polymorphism and susceptibility to malaria, NO levels or parasitaemia. Indeed, the G-954-C C allele was present in less than $4 \%$ of the study population, it is absent in Caucasian and is found at low frequency in Asia. [26]. In contrast, in African population this genotype is present in high frequency where most of the associations with malaria outcome were reported. Even though, there has been much speculation about the role played by nitric oxide (NO) in malaria, both as an antiparasitic agent and as a potential cause of cerebral malaria [37]. In Brazil, the NOS2A-954G/C polymorphism have been reported in studies with tuberculosis and leprosy. In both studies, the allelic and genotypic frequencies were similar to the one found in this study, even though their population were from South and Southeast region [50,51]. Although no association has been found, this study is the first to report NOS2A-954G/C polymorphism and NO levels in malaria exposed individuals in endemic region of Brazil. Indeed, the NOS $2 \mathrm{~A}$ gene polymorphism have been associated with susceptibility to $P$. falciparum malaria and conflicting results have been obtained in studies that associate the presence of the G-954-C C allele and either risk of cerebral malaria or NO production [26,52]. It should be noted that individual differences in the levels of the cytokines and $\mathrm{NO}$ measured at a specific moment may not only result from host genetic factors predisposing to high or low production, but also for a great part from the physiological condition at that time, as well as from general immunity. The present findings reinforce the role of mediators of inflammation in malaria susceptibility and future studies in different setting with large samples numbers are warranted. Furthermore, it should be observed that not merely one genetic alteration but rather the combination of a set of genetic factors might influence the susceptibility or resistance to malaria.

\section{Conclusions}

This study shows that IFNG $+874 T / A, I L 10 A-1082 G / A$ and NOS2A-954G/C polymorphisms was not associated with the occurrence of malaria or with the production of its respective cytokine and nitric oxide products. The IL10A-592A/C and IL10A-819 T/C polymorphisms were associated with malaria and decreased IL-10 levels and low parasite density suggesting that this polymorphism influence IL-10 levels and may influence in the susceptibility to clinical malaria in Amazonian population.

\footnotetext{
Abbreviations

SNP: Single nucleotide polymorphism; PCR: Polymerase chain reaction; DNA: Deoxyribonucleic acid; IFN-y: Interferon-gamma; NO: Nitric oxide; TNF: Tumour necrosis factor; IL-10: Interleukin 10; iNOS: Enzyme nitric oxide synthase; NADPH: $\beta$-nicotinamide adenine dinucleotide phosphate; OR: Odds ratio; Cl: Confidence interval; HWE: Hardy Weinberg equilibrium; EM: Expectation Maximization; E: Hope; M: Max.
} 


\section{Competing interests}

The authors declare that they have no competing interests.

\section{Authors' contributions}

JOF conceived the idea for this study. JOF, MGBA and JCLJ designed the study. Fieldwork and sample collection was done by JCSA, DSPS, MPAV, CAML, CJLA, DMB and AT. VAP, JCSA, DSPS, RNRS and DOS performed the experiments. Data was collected and analysed by VAP with support from JCSA, MGBA, JOF and JCLJ. The first draft of this manuscript was written by VAP and JOF and MGBA critically read and advised on the manuscript. All authors read and approved the final version.

\section{Acknowledgements}

This work was supported by PRONEX Malaria/CNPq/FAPERJ. JOF is recipient of Research Productivity Fellowships from the Conselho Nacional de Desenvolvimento Científico e Tecnológico (CNPq). VAR and JCSA are recipients of a fellowship from $\mathrm{CNPq}$ and Instituto Oswaldo Cruz, respectively. We thank the Secretary of Health of Rondônia State, the Laboratório Central - LACEN of Rondônia for fieldwork support. We are grateful to all individuals that participated in this study for their cooperation and generous donation of blood, which made this study possible.

\section{Author details}

${ }^{1}$ Laboratório de Imunoparasitologia, Instituto Oswaldo Cruz/Fiocruz, Av. Brasil 4365, Manguinhos, Rio de Janeiro, Brazil. 'Laboratório de Imunodiagnóstico /Departamento de Ciências Biológicas, Escola Nacional de Saúde Pública/ Fiocruz, Rio de Janeiro, Rio de Janeiro, Brazil. ${ }^{3}$ Laboratório de Simulídeos e Oncocercose, Instituto Oswaldo Cruz/Fiocruz, Rio de Janeiro, Brazil. ${ }^{4}$ Instituto de Infectologia Emilio Ribas, São Paulo, São Paulo, Brazil. ${ }^{5}$ Centro Interdepartamental de Biologia Experimental e Biotecnologia, Universidade Federal de Rondônia, Porto Velho, Rondônia, Brazil. 'Laboratório de Quimioterapia/Fiocruz, Porto Velho, Rondônia, Brazil and Universidade Federal de Rondônia, Porto Velho, Rondônia, Brazil. 'Laboratório de Imunologia e Imunogenética, Instituto de Pesquisa Clínica Evandro Chagas (IPEC)/Fiocruz, Rio de Janeiro, Rio de Janeiro, Brazil.

Received: 8 August 2014 Accepted: 6 January 2015

Published online: 28 January 2015

\section{References}

1. Luzzatto L. Genetic factors in malaria. Bull World Health Organ. 1974;50:195-202.

2. Driss A, Hibbert JM, Wilson NO, Iqbal SA, Adamkiewicz TV, Stiles JK. Genetic polymorphisms linked to susceptibility to malaria. Malar J. 2011;10:271.

3. Clark IA, Budd AC, Alleva LM, Cowden WB. Human malarial disease: a consequence of inflammatory cytokine release. Malar J. 2006;5:85.

4. Andrade BB, Reis-Filho A, Souza-Neto SM, Clarencio J, Camargo LM, Barral A et al. Severe Plasmodium vivax malaria exhibits marked inflammatory imbalance. Malar J. 2010;9:13.

5. Akanmori BD, Kurtzhals JA, Goka BQ, Adabayeri V, Ofori MF, Nkrumah FK, et al. Distinct patterns of cytokine regulation in discrete clinical forms of Plasmodium falciparum malaria. Eur Cytokine Netw. 2000;11:113-8.

6. Kern P, Hemmer CJ, Van Damme J, Gruss HJ, Dietrich M. Elevated tumor necrosis factor alpha and interleukin-6 serum levels as markers for complicated Plasmodium falciparum malaria. Am J Med. 1989;87:139-43.

7. Hugosson E, Montgomery SM, Premji Z, Troye-Blomberg M, Bjorkman A. Higher IL-10 levels are associated with less effective clearance of Plasmodium falciparum parasites. Parasite Immunol. 2004:26:111-7.

8. Lopansri BK, Anstey NM, Weinberg JB, Stoddard GJ, Hobbs MR, Levesque $M C$, et al. Low plasma arginine concentrations in children with cerebral malaria and decreased nitric oxide production. Lancet. 2003;361:676-8.

9. Wilson NO, Bythwood T, Solomon W, Jolly P, Yatich N, Jiang Y, Shuaib F, Adjei AA, Anderson W, Stiles JK. Elevated levels of IL-10 and G-CSF associated with asymptomatic malaria in pregnant women. Infect Dis Obstet Gynecol. 2010;2010.

10. Rodrigues-da-Silva RN, Lima-Junior J d C, Fonseca BP, Antas PR, Baldez A, Storer $\mathrm{FL}$, et al. Alterations in cytokines and haematological parameters during the acute and convalescent phases of Plasmodium falciparum and Plasmodium vivax infections. Mem Inst Oswaldo Cruz. 2014;109:154-62.

11. Pacheco AG, Cardoso CC, Moraes MO. IFNG +874 T/A, IL10-1082G/A and TNF -308G/A polymorphisms in association with tuberculosis susceptibility: a meta-analysis study. Hum Genet. 2008;123:477-84.
12. Matos Gl, Covas Cde J, Bittar Rde C, Gomes-Silva A, Marques F, Maniero VC, et al. IFNG +874 T/A polymorphism is not associated with American tegumentary leishmaniasis susceptibility but can influence Leishmania induced IFN-gamma production. BMC Infect Dis. 2007;7:33.

13. Vallinoto AC, Graca ES, Araujo MS, Azevedo VN, Cayres-Vallinoto I, Machado LF, et al. IFNG +874 T/A polymorphism and cytokine plasma levels are associated with susceptibility to Mycobacterium tuberculosis infection and clinical manifestation of tuberculosis. Hum Immunol. 2010;71:692-6.

14. Cardoso CC, Pereira AC, Brito-de-Souza VN, Dias-Baptista IM, Maniero VC, Venturini J, et al. IFNG +874 T > A single nucleotide polymorphism is associated with leprosy among Brazilians. Hum Genet. 2010;128:481-90.

15. Ribeiro CS, Visentainer JE, Moliterno RA. Association of cytokine genetic polymorphism with hepatitis B infection evolution in adult patients. Mem Inst Oswaldo Cruz. 2007;102:435-40.

16. Koch O, Rockett K, Jallow M, Pinder M, Sisay-Joof F, Kwiatkowski D. Investigation of malaria susceptibility determinants in the IFNG/IL26/IL22 genomic region. Genes Immun. 2005;6:312-8.

17. Cabantous S, Poudiougou B, Traore A, Keita M, Cisse MB, Doumbo O, et al. Evidence that interferon-gamma plays a protective role during cerebral malaria. J Infect Dis. 2005;192:854-60.

18. Medina TS, Costa SP, Oliveira MD, Ventura AM, Souza JM, Gomes TF, et al. Increased interleukin-10 and interferon-gamma levels in Plasmodium vivax malaria suggest a reciprocal regulation which is not altered by IL-10 gene promoter polymorphism. Malar J. 2011;10:264.

19. Giordani L, Bruzzi P, Lasalandra C, Quaranta M, Schittulli F, Della Ragione F, et al. Association of breast cancer and polymorphisms of interleukin-10 and tumor necrosis factor-alpha genes. Clin Chem. 2003;49:1664-7.

20. Ouma C, Davenport GC, Were T, Otieno MF, Hittner JB, Vulule JM, et al Haplotypes of IL-10 promoter variants are associated with susceptibility to severe malarial anemia and functional changes in IL-10 production. Hum Genet. 2008;124:515-24.

21. Jason J, Archibald LK, Nwanyanwu OC, Bell M, Buchanan I, Larned J, et al. Cytokines and malaria parasitemia. Clin Immunol. 2001;100:208-18.

22. Keller CC, Davenport GC, Dickman KR, Hittner JB, Kaplan SS, Weinberg JB, et al. Suppression of prostaglandin E2 by malaria parasite products and antipyretics promotes overproduction of tumor necrosis factor-alpha: association with the pathogenesis of childhood malarial anemia. J Infect Dis. 2006;193:1384-93.

23. May J, Lell B, Luty AJ, Meyer CG, Kremsner PG. HLA-DQB1*0501-restricted Th1 type immune responses to Plasmodium falciparum liver stage antigen 1 protect against malaria anemia and reinfections. J Infect Dis. 2001;183:168-72.

24. Clark IA, Cowden WB, Rockett KA. Nitric oxide in cerebral malaria. J Infect Dis. 1995;171:1068-9.

25. el-Nashar TM, el-Kholy HM, el-Shiety AG, Al-Zahaby AA. Correlation of plasma levels of tumor necrosis factor, interleukin-6 and nitric oxide with the severity of human malaria. J Egypt Soc Parasitol. 2002;32:525-35.

26. Kun JF, Mordmuller B, Perkins DJ, May J, Mercereau-Puijalon O, Alpers M, et al. Nitric oxide synthase 2(Lambarene) (G-954C), increased nitric oxide production, and protection against malaria. J Infect Dis. 2001;184:330-6.

27. Anstey NM, Granger DL, Hassanali MY, Mwaikambo ED, Duffy PE, Weinberg JB. Nitric oxide, malaria, and anemia: inverse relationship between nitric oxide production and hemoglobin concentration in asymptomatic, malaria-exposed children. Am J Trop Med Hyg. 1999;61:249-52.

28. Kun JF, Mordmuller B, Lell B, Lehman LG, Luckner D, Kremsner PG. Polymorphism in promoter region of inducible nitric oxide synthase gene and protection against malaria. Lancet. 1998;351:265-6.

29. Dhangadamajhi G, Mohapatra BN, Kar SK, Ranjit MR. A new allele (eNOS4e) in the intron 4 (VNTR) of eNOS gene in malaria infected individuals of the population of Orissa (an eastern Indian state). Nitric Oxide. 2010;22:58-9.

30. Jha AN, Singh VK, Kumari N, Singh A, Antony J, van Tong $H$, et al. IL-4 haplotype -590 T, -34 T and intron-3 VNTR R2 is associated with reduced malaria risk among ancestral indian tribal populations. PLoS One. 2012;7:e48136.

31. Sortica VA, Cunha MG, Ohnishi MD, Souza JM, Ribeiro-Dos-Santos AK, Santos $N P$, et al. IL1B, IL4R, IL12RB1 and TNF gene polymorphisms are associated with Plasmodium vivax malaria in Brazil. Malar J. 2012;11:409.

32. Santos SD, Clark TG, Campino S, Suarez-Mutis MC, Rockett KA, Kwiatkowski $\mathrm{DP}$, et al. Investigation of host candidate malaria-associated risk/protective SNPs in a Brazilian Amazonian population. PLoS One. 2012;7:e36692. 
33. Snounou G, Viriyakosol S, Zhu XP, Jarra W, Pinheiro L, do Rosario VE, et al. High sensitivity of detection of human malaria parasites by the use of nested polymerase chain reaction. Mol Biochem Parasitol. 1993;61:315-20.

34. Saúde MD, Saúde SV. Guia Prático de Tratamento da Malária no Brasil. Brasília-DF: Annual Report; 2010.

35. Newton CR, Graham A, Heptinstall LE, Powell SJ, Summers C, Kalsheker N, et al. Analysis of any point mutation in DNA. The amplification refractory mutation system (ARMS). Nucleic Acids Res. 1989;17:2503-16.

36. Rockett KA, Awburn MM, Rockett EJ, Clark IA. Tumor necrosis factor and interleukin-1 synergy in the context of malaria pathology. Am J Trop Med Hyg. 1994;50:735-42

37. Nahrevanian H, Dascombe MJ. Nitric oxide and reactive nitrogen intermediates during lethal and nonlethal strains of murine malaria. Parasite Immunol. 2001;23:491-501.

38. Bidwell J, Keen L, Gallagher G, Kimberly R, Huizinga T, McDermott MF, et al Cytokine gene polymorphism in human disease: on-line databases, supplement 1. Genes Immun. 2001;2:61-70.

39. Rodriguez Lopez R. [Human susceptibility to complex diseases. Genetic analysis in large populations](in Portuguese). Rev Derecho Genoma Hum. 2004;227-239.

40. Hill AV. Aspects of genetic susceptibility to human infectious diseases. Annu Rev Genet. 2006;40:469-86

41. Torres OA, Calzada JE, Beraun Y, Morillo CA, Gonzalez A, Gonzalez Cl, et al. Role of the IFNG +874 T/A polymorphism in chagas disease in a Colombian population. Infect Genet Evol. 2010;10:682-5.

42. Mangano VD, Clark TG, Auburn S, Campino S, Diakite M, Fry AE, et al. Lack of association of interferon regulatory factor 1 with severe malaria in affected child-parental trio studies across three African populations. PLoS One. 2009;4:e4206

43. Naka I, Patarapotikul J, Hananantachai H, Tokunaga K, Tsuchiya N, Ohashi J. IFNGR1 polymorphisms in Thai malaria patients. Infect Genet Evol. 2009:9:1406-9.

44. Turner DM, Williams DM, Sankaran D, Lazarus M, Sinnott PJ, Hutchinson IV. An investigation of polymorphism in the interleukin-10 gene promoter. Eur J Immunogenet. 1997;24:1-8.

45. Kariuki SM, Rockett K, Clark TG, Reyburn H, Agbenyega T, Taylor TE, et al. The genetic risk of acute seizures in African children with falciparum malaria. Epilepsia. 2013;54:990-1001.

46. Zhang G, Manaca MN, McNamara-Smith M, Mayor A, Nhabomba A, Berthoud TK, et al. Interleukin-10 (IL-10) polymorphisms are associated with IL-10 production and clinical malaria in young children. Infect Immun. 2012;80:2316-22

47. Ohashi J, Naka I, Patarapotikul J, Hananantachai H, Looareesuwan S, Tokunaga K. Lack of association between interleukin-10 gene promoter polymorphism, $-1082 \mathrm{G} / \mathrm{A}$, and severe malaria in Thailand. Southeast Asian J Trop Med Public Health. 2002;33 Suppl 3:5-7.

48. Lins TC, Vieira RG, Abreu BS, Grattapaglia D, Pereira RW. Genetic composition of Brazilian population samples based on a set of twenty-eight ancestry informative SNPs. Am J Hum Biol. 2010;22:187-92.

49. Wilson JN, Rockett K, Jallow M, Pinder M, Sisay-Joof F, Newport M, et al. Analysis of IL10 haplotypic associations with severe malaria. Genes Immun. 2005:6:462-6.

50. Leandro AC, Rocha MA, Lamoglia-Souza A, VandeBerg UL, Rolla VC, Bonecini-Almeida Mda G. No association of IFNG +874 T/A SNP and NOS2A-954G/C SNP variants with nitric oxide radical serum levels or susceptibility to tuberculosis in a Brazilian population subset. Biomed Res Int. 2013:2013:901740.

51. Messias-Reason IJT, van Tong H, Velavan TP. Analysis of polymorphic sites in the promoter of the nitric oxide synthase 2 gene in Brazilian patients with leprosy. Int J Immunogenet. 2014;41:231-5.

52. Ohashi J, Naka I, Patarapotikul J, Hananantachai H, Looareesuwan S, Tokunaga K. Significant association of longer forms of CCTTT Microsatellite repeat in the inducible nitric oxide synthase promoter with severe malaria in Thailand. J Infect Dis. 2002:186:578-81.

\section{Submit your next manuscript to BioMed Central and take full advantage of:}

- Convenient online submission

- Thorough peer review

- No space constraints or color figure charges

- Immediate publication on acceptance

- Inclusion in PubMed, CAS, Scopus and Google Scholar

- Research which is freely available for redistribution

Submit your manuscript at www.biomedcentral.com/submit 